\title{
Existence of new inequalities for representable polymatroids
}

\author{
Terence Chan, Alex Grant and Doris Kern
}

\begin{abstract}
An Ingletonian polymatroid satisfies, in addition to the polymatroid axioms, the inequalities of Ingleton (Combin. Math. Appln., 1971). These inequalities are required for a polymatroid to be representable. It is has been an open question as to whether these inequalities are also sufficient. Representable polymatroids are of interest in their own right. They also have a strong connection to network coding. In particular, the problem of finding the linear network coding capacity region is equivalent to the characterization of all representable, entropic polymatroids. In this paper, we describe a new approach to adhere two polymatroids together to produce a new polymatroid. Using this approach, we can construct a polymatroid that is not inside the minimal closed and convex cone containing all representable polymatroids. This polymatroid is proved to satisfy not only the Ingleton inequalities, but also the recently reported inequalities of Dougherty, Freiling and Zeger. A direct consequence is that these inequalities are not sufficient to characterize representable polymatroids.
\end{abstract}

\section{INTRODUCTION}

The idea of network coding was first proposed in the seminal paper [1] as a means to increase achievable transmission throughput in data communications networks. In the traditional packetswitched routing approach, intermediate network nodes can only duplicate received packets and forward them to subsequent nodes. In contrast, network coding allows arbitrary computational data processing at intermediate nodes. For example, intermediate nodes may forward arbitrary linear combinations of several received packets. In the single source multicast scenario, network coding significantly increases maximal transmission throughput, and achieves the max-flow mincut bound. It was subsequently proved [2] that linear network codes suffice to achieve maximal throughput for this case. 
While the easily computable maximum flow (and associated minimum cut) determines the maximal attainable throughput in the single source scenario, this bound is not tight in general (multiple sources and multiple sinks). In [3], first steps were made to characterize transmission throughput for the general case via entropy functions (polymatroids whose ground set is a set of random variables, and whose rank function is Shannon entropy). Inner and outer bounds on throughput were obtained in this way. Using the same idea, an exact characterization of the set of all achievable throughputs was later obtained [4]. Analogous bounds for networks where intermediate nodes are restricted to use only linear codes were obtained in [5] via representable entropy functions.

Unfortunately, these entropy function based characterizations are implicit in nature, since an explicit characterization of the set of all entropy functions is still missing. Characterization of this set is one of the major open problems in information theory. Similarly, the set of all representable entropy functions has no explicit characterization. Notably, this set is a subset of representable polymatroids, whose characterization is one of the major open problems in matroid theory.

This lack of explicit, computable results could prompt one to question this approach based on entropy functions. Although it leads to attractive implicit characterizations, perhaps the difficulties that arise are somehow an artefact of the approach. One could therefore be tempted to seek simpler characterizations of transmission throughput that avoid the need to precisely know the set of entropy functions. For instance, [1] demonstrated that a much simpler characterization is possible in the single source scenario where the max-flow min-cut bound is tight. Unfortunately, a recent paper [6] disproved the existence of any simpler characterization for the general case. Using a specially contructed network, it was proved that if one can determine the set of all achievable throughputs in the special network, then one can also determine the set of all entropy functions (and vice-versa). Hence, determining achievable throughput for network coding is in general no simpler than determining the set of entropy functions. A similar duality was obtained in the same paper between the set of throughputs achieved by linear codes and the set of representable entropy functions.

These results [3]-[6] indicate a very close tie between characterization of (representable) entropy functions and throughput achievable with (linear) network codes. Characterization of entropy functions is equivalent to finding all linear information inequalities that hold regardless of 
the underlying joint distribution [3]. It is a well known result, extending back to Shannon [7] that entropy and mutual information are both nonnegative, correpsonding exactly to the polymatroid axioms. No further information inequalities were found for fifty years, until [8] reported the first "non-Shannon" information inequality. The significance of that result lay not only in the inequality itself, but also in its construction. This particular approach for construction has been the main ingredient in every non-Shannon inequality that has been subsequently discovered. Using this appraoch, new inequalities can be found mechanically [9] and there are in fact infinitely many such independent inequalities even when there are only four random variables involved [10]. Despite this progress, a complete characterization is still missing, and we still only have one basic approach for finding new inequalities.

This situation does not improve for representable entropy functions. In addition to the polymatroid inequalities, it is well known that representable entropy functions satisfy Ingleton's inequalities [11]. Specifically, let $W_{1}, \ldots, W_{4}$ be vector subspaces. Then

$$
\begin{aligned}
0 \leq & \operatorname{dim}\left\langle W_{1}, W_{2}\right\rangle+\operatorname{dim}\left\langle W_{1}, W_{3}\right\rangle+\operatorname{dim}\left\langle W_{1}, W_{4}\right\rangle+\operatorname{dim}\left\langle W_{2}, W_{3}\right\rangle+\operatorname{dim}\left\langle W_{2}, W_{4}\right\rangle \\
& \quad-\operatorname{dim}\left\langle W_{1}\right\rangle-\operatorname{dim}\left\langle W_{2}\right\rangle-\operatorname{dim}\left\langle W_{3}, W_{4}\right\rangle-\operatorname{dim}\left\langle W_{1}, W_{2}, W_{3}\right\rangle-\operatorname{dim}\left\langle W_{1}, W_{2}, W_{4}\right\rangle
\end{aligned}
$$

where $\left\langle W_{i}, W_{j}\right\rangle$ is the minimal vector subspace containing $W_{i} \cup W_{j}$, and similar for $\left\langle W_{i}, W_{j}, W_{k}\right\rangle$. It has been an open problem since 1971 as to whether these inequalities are also sufficient as well as being necessary conditions for representability.

Very recently, several new inequalities for representable polymatroids were reported at the 2009 Workshop on Applications of Matroid Theory and Combinatorial Optimization to Information and Coding Theory [1211. These inequalities were found by adapting the approach in [8], [9]. It was verified numerically that the newly obtained inequalities (which we shall refer to as $D F Z$ inequalities) completely characterize representable entropy functions involving five variables (the Ingleton inequalities are already known to be sufficient for four variables). It is not known if these inequalities remain sufficient for more than five variables.

The objective of this paper is to understand properties of representable entropy functions (and more generally, representable polymatroids). Our main contribution is a proof for the insufficiency of the Ingleton and DFZ inequalities for charcterization of representable polymatroids.

\footnotetext{
${ }^{1}$ We became aware of this independent work during the preparation of early drafts of this manuscript.
} 
Whereas [12] constructively proves the insufficiency of the Ingleton inequalities following the Zhang-Yeung approach [8], our approach is totally different. We construct a polymatroid which satisfies every Ingleton and DFZ inequality, but which is not contained within the minimal closed and convex cone containing all representable polymatroids. This directly establishes the existence of further, yet-to-be-discovered linear inequalities for representable polymatroids.

The organization of the paper is as follows. In Section II, we will introduce the required technical framework for the problem. Section [II introduces a new method for constructing a polymatroid by adhering together any two Ingleton polymatroids. We will prove that the resulting polymatroids satisfy the Ingleton inequalities, and that this construction also preserves representability. In Section $\nabla$, we will construct an Ingleton polymatroid by adhering two representable polymatroids together. This constructed polymatroid will be proved in Section IV] to lie outside the closed and convex cone containing all representable polymatroids. This establishes the insufficiency of Ingleton's inequalities. Finally, in Section VI, we prove that this constructed polymatroid also satisfies the DFZ inequalities (for five variables), demonstrating the insufficiency of the DFZ inequalities.

The following notational conventions will be used. Set union will be denoted by concatenation; Singletons and sets with one element are not distinguished; Given $\mathcal{X}=\left\{X_{1}, X_{2}, \ldots, X_{n}\right\}$ and any subset $\alpha$ of the finite index set $\mathcal{N}_{n}=\{1,2, \ldots, n\}$, the subscript $X_{\alpha}$ will mean the set $\left\{X_{i}, i \in \alpha\right\}$. For $\alpha, \beta \subseteq \mathcal{N}_{n}, X_{\alpha \beta}=X_{\alpha} X_{\beta}=X_{\alpha} \cup X_{\beta}$ all refer to the same set. Similarly, for any $\mathcal{A}, \mathcal{B} \subseteq \mathcal{X}, \mathcal{A} \cup \mathcal{B}$ and $\mathcal{A B}$ are the same set. $\langle S\rangle$ will denote the minimal vector space spanned by $S$. We will use $\operatorname{con}(\mathcal{S})$ to denote the minimal convex cone containing the set $\mathcal{S}$ and $\overline{\operatorname{con}}(\mathcal{S})$ to denote the closure of $\operatorname{con}(\mathcal{S})$. Finally, $\mathbb{R}, \mathbb{Z}$ and $\mathbb{F}_{q}$ are the reals, integers and a finite field on $q$ elements.

\footnotetext{
${ }^{2}$ If $n$ is understood, the subscript may be dropped for simplicity.
} 


\section{BACKGROUND}

A polymatroid over the ground set $\mathcal{X}=\left\{X_{1}, X_{2}, \ldots, X_{n}\right\}$ is a tuple $(\mathcal{X}, \mathbf{h})$ where the rank function $\mathbf{h}: 2^{\mathcal{X}} \mapsto \mathbb{R}_{+}$satisfies the following axioms for all $\mathcal{A}, \mathcal{B} \subseteq \mathcal{X}$,

$$
\begin{aligned}
& \mathbf{h}(\emptyset)=0 \\
& \mathcal{A} \subseteq \mathcal{B} \Longrightarrow \mathbf{h}(\mathcal{A}) \leq \mathbf{h}(\mathcal{B}) \\
& \mathbf{h}(\mathcal{A} \cup \mathcal{B})+\mathbf{h}(A \cap \mathcal{B}) \leq \mathbf{h}(A)+\mathbf{h}(\mathcal{B}) .
\end{aligned}
$$

A polymatroid $(\mathcal{X}, \mathbf{h})$ is called a matroid [13], if it further satisfies the cardinality bound, $\mathbf{h}(\mathcal{A}) \leq|\mathcal{A}|$, and the integrality constraint $\mathbf{h}(\mathcal{A}) \in \mathbb{Z}$, for all $\mathcal{A}, \mathcal{B} \subseteq \mathcal{X}$.

For any $\mathcal{A}, \mathcal{B}, \mathcal{C} \subseteq \mathcal{X}$, define the generalized information expressions as follows:

$$
\begin{aligned}
H(\mathcal{A} \mid \mathcal{C}) & \triangleq \mathbf{h}(\mathcal{A C})-\mathbf{h}(\mathcal{C}) \\
I(\mathcal{A} ; \mathcal{B} \mid \mathcal{C}) & \triangleq \mathbf{h}(\mathcal{A C})+\mathbf{h}(\mathcal{B C})-\mathbf{h}(\mathcal{C})-\mathbf{h}(\mathcal{A B C})
\end{aligned}
$$

when $\mathcal{C}=\emptyset$ we write $H(\mathcal{A})=\mathbf{h}(\mathcal{A})$ and $I(\mathcal{A} ; \mathcal{B})=H(\mathcal{A})-H(\mathcal{A} \mid \mathcal{B})$ (consistent with the above definitions). It is straightforward to prove that $(\mathcal{X}, \mathbf{h})$ is a polymatroid if and only if (R1) holds and both (2) and (3) are nonnegative for all choices of $\mathcal{A}, \mathcal{B}$ and $\mathcal{C}$.

Polymatroids arise in many different contexts. For example, let $\mathcal{X}=\left\{X_{1}, \ldots, X_{n}\right\}$ be a set of random variables. This naturally induces a polymatroid $(\mathcal{X}, \mathbf{h})$ such that $\mathbf{h}(\mathcal{A})$ is the Shannon entropy $H(\mathcal{A})$ of the subset of random variables in $\mathcal{A}$. In this case, (2) and (3) are merely the usual definitions for conditional entropy and mutual information, and $(\mathcal{X}, \mathbf{h})$ is a polymatroid due to the nonnegativity of (conditional) entropies and mutual information. We emphasise however that the definitions (2) and (3) are made for arbitrary polymatroids (where the rank function may not induced by random variables).

Polymatroids can also be induced by vector subspaces. Let $\mathcal{X}=\left(X_{1}, \ldots, X_{n}\right)$ be a set of subspaces of a vector space $\mathbb{W}$ over a finite field $\mathbb{F}_{q}$. Define $\mathbf{h}(\mathcal{A})$ as the dimension of the minimal vector subspace containing all the subspaces in $\mathcal{A}$,

$$
\mathbf{h}(\mathcal{A}) \triangleq \operatorname{dim}\langle\mathcal{A}\rangle
$$

Then $(\mathcal{X}, \mathbf{h})$ is also a polymatroid. These subspace induced polymatroids (called representable polymatroids) are of the main objects of interest in this paper. 
According to definitions (2) and (3) above, when $\mathbf{h}$ is defined as in (4), we can use $H(\mathcal{A})$ to denote $\operatorname{dim}\langle\mathcal{A}\rangle$ for any set of vector subspaces $\mathcal{A}$. Furthermore, the following lemma may be easily verified.

Lemma 1: Let $\mathcal{X}=\left(X_{1}, \ldots, X_{n}\right)$ be a set of vector subspaces and $\mathcal{A}, \mathcal{B} \subseteq \mathcal{X}$. Then

$$
\begin{aligned}
& H(\mathcal{A} \mid \mathcal{B})=\operatorname{dim}\langle\mathcal{A}, \mathcal{B}\rangle-\operatorname{dim}\langle\mathcal{B}\rangle \\
& I(\mathcal{A} ; \mathcal{B})=\operatorname{dim}(\langle\mathcal{A}\rangle \cap\langle\mathcal{B}\rangle)
\end{aligned}
$$

Furthermore, if $V=\langle\mathcal{A}\rangle \cap\langle\mathcal{B}\rangle$, then $I(\mathcal{A} ; \mathcal{B} \mid V)=0$.

We shall classify polymatroids as follows.

Definition 1 (Classification): A polymatroid $(\mathcal{X}, \mathbf{h})$, and associated rank function $\mathbf{h}$, is called

- q-representable if there exists vector subspaces $\left\{V_{1}, \ldots, V_{n}\right\}$ over $\mathbb{F}_{q}$ such that for all $\alpha \subseteq$ $\mathcal{N}_{n}, H\left(X_{\alpha}\right)=\operatorname{dim}\left\langle V_{\alpha}\right\rangle$ as defined in (4).

- representable if is $q$-representable for some $q$

- even representable if is $2^{m}$-representable for some positive integer $m$

- odd representable if is $p^{m}$-representable for some odd prime $p$ and a positive integer $m$

- Ingletonian if it satisfies the Ingleton inequality $J_{\mathbf{h}}\left(\mathcal{A}_{1}, \mathcal{A}_{2}, \mathcal{A}_{3}, \mathcal{A}_{4}\right) \geq 0$ for all subsets $\mathcal{A}_{1}, \mathcal{A}_{2}, \mathcal{A}_{3}, \mathcal{A}_{4} \subseteq \mathcal{X}$ where

$$
\begin{aligned}
J_{\mathbf{h}}\left(\mathcal{A}_{1}, \mathcal{A}_{2}, \mathcal{A}_{3}, \mathcal{A}_{4}\right) \triangleq & \mathbf{h}\left(\mathcal{A}_{1} \mathcal{A}_{2}\right)+\mathbf{h}\left(\mathcal{A}_{1} \mathcal{A}_{3}\right)+\mathbf{h}\left(\mathcal{A}_{1} \mathcal{A}_{4}\right)+\mathbf{h}\left(\mathcal{A}_{2} \mathcal{A}_{3}\right)+\mathbf{h}\left(\mathcal{A}_{2} \mathcal{A}_{4}\right) \\
& -\mathbf{h}\left(\mathcal{A}_{1}\right)-\mathbf{h}\left(\mathcal{A}_{2}\right)-\mathbf{h}\left(\mathcal{A}_{3} \mathcal{A}_{4}\right)-\mathbf{h}\left(\mathcal{A}_{1} \mathcal{A}_{2} \mathcal{A}_{3}\right)-\mathbf{h}\left(\mathcal{A}_{1} \mathcal{A}_{2} \mathcal{A}_{4}\right)
\end{aligned}
$$

According to [11], if a polymatroid $(\mathcal{X}, \mathbf{h})$ is representable, then it is also Ingletonian. A natural question then arises - Are the Ingleton inequalities sufficient to characterize representable polymatroids? In this paper, we will show that the answer to this question is negative.

Following the framework for information inequalities presented in [14], it is useful to treat a rank function as a vector or point in a $2^{|\mathcal{X}|}$-dimensional real Euclidean space whose coordinates are indexed by the power set of $\mathcal{X}$. Thus a point $\mathbf{h} \in \mathbb{R}^{2^{|\mathcal{X}|}}$ is specified by its coordinates as $\mathbf{h}=(\mathbf{h}(\mathcal{A}): \mathcal{A} \subseteq \mathcal{X})$. While a polymatroid is defined by a ground set and a rank function, the ground set is actually implicitly defined by the rank function. Hence, strictly speaking, a polymatroid $(\mathcal{X}, \mathbf{h})$ is no more than a rank function that satisfies the polymatroidal axioms. 
In other words, a polymatroid is merely a point in an Euclidean space and characterization of representable polymatroids is equivalent to characterizing those points induced by representable polymatroids.

Regarding polymatroids as points in $\mathbb{R}^{2^{|\mathcal{X}|}}$ permits us to define metrics and limits on the set of polymatroids. Let $\Upsilon_{q}[\mathcal{X}]$ be the set of all $q$-representable rank functions and $\left.\Upsilon[\mathcal{X}]=\bigcup_{q} \Upsilon_{q}[\mathcal{X}]\right)$ be the set of all representable rank functions.

Definition 2: A polymatroid $(\mathcal{X}, \mathbf{h}$ ) (and the corresponding rank function $\mathbf{h}$ ) is called almost representable if there exists a sequence of representable rank functions $\left\{\mathbf{g}_{i}\right\}_{i=1}^{\infty}$ and a sequence of positive numbers $c_{i}$ such that $\mathbf{h}=\lim _{i \rightarrow \infty} c_{i} \mathbf{g}_{i}$. On the other hand, $\mathbf{h}$ is called cc-representable 3 if $\mathbf{h} \in \overline{\operatorname{con}}(\Upsilon[\mathcal{X}])$.

A linear inequality involving polymatroids is merely a linear inequality over $\mathbb{R}^{\mid \mathcal{| X |}}$. We are interested to determine necessary conditions (linear inequalities in particular) on the rank function $\mathbf{h}$ under which it is representable. In this paper, complete characterization of representable rank functions means an explicit determination of $\overline{\operatorname{con}}(\Upsilon[\mathcal{X}])$ or the set of linear inequalities satisfied by points in $\overline{\operatorname{con}}(\Upsilon[\mathcal{X}])$. The following proposition may be directly verified.

Proposition 1: A linear inequality $\sum_{i} c_{i} H(\mathcal{A}) \geq 0$ holds for all representable polymatroids (i.e. is a valid subspace rank inequality) if and only if $\sum_{i} c_{i} \mathbf{h}(\mathcal{A}) \geq 0$ for all $\mathbf{h} \in \overline{\operatorname{con}}(\Upsilon[\mathcal{X}])$.

\section{CREATING NEW POLYMATROIDS}

We now propose an approach to perturb an Ingletonian polymatroid in a way that preserves the Ingletonian property. This is achieved by Theorem 11. We shall subsequently show in Theorems 2 and 3 that this perturbation also preserves (almost) representability. In Section IV] we will use this approach to perturb a member of $\overline{\operatorname{con}}(\Upsilon[\mathcal{X}])$, taking it outside of $\overline{\operatorname{con}}(\Upsilon[\mathcal{X}])$. This perturbed polymatroid will be used to show the existence of new subspace rank inequalities for representable polymatroids.

Theorem 1 ( $\epsilon$-pertubation): Let $(\mathcal{Y}, \mathbf{h})$ be an Ingletonian polymatroid. Let $0 \leq \epsilon \leq \mathbf{h}(\mathcal{Y})$ and define for all $\mathcal{A} \subseteq \mathcal{Y}$

$$
\mathbf{g}(\mathcal{A}) \triangleq \min (\mathbf{h}(\mathcal{A}), \mathbf{h}(\mathcal{Y})-\epsilon)
$$

3 "cc" is a mnemonic for "Closed and Convex cone". 
Then $(\mathcal{Y}, \mathbf{g})$ is also an Inlgetonian polymatroid.

Proof: We need to prove that

$$
J_{\mathrm{g}}\left(\mathcal{V}_{1}, \mathcal{V}_{2}, \mathcal{V}_{3}, \mathcal{V}_{4}\right) \geq 0
$$

for all $\mathcal{V}_{1}, \ldots, \mathcal{V}_{4} \subseteq \mathcal{Y}$. To simplify notation, define

$$
\begin{aligned}
& J_{\mathrm{g}}^{+}\left(\mathcal{V}_{1}, \mathcal{V}_{2}, \mathcal{V}_{3}, \mathcal{V}_{4}\right) \triangleq \mathbf{g}\left(\mathcal{V}_{1} \mathcal{V}_{2}\right)+\mathbf{g}\left(\mathcal{V}_{1} \mathcal{V}_{3}\right)+\mathbf{g}\left(\mathcal{V}_{1} \mathcal{V}_{4}\right)+\mathbf{g}\left(\mathcal{V}_{2} \mathcal{V}_{3}\right)+\mathbf{g}\left(\mathcal{V}_{2} \mathcal{V}_{4}\right) \\
& J_{\mathrm{g}}^{-}\left(\mathcal{V}_{1}, \mathcal{V}_{2}, \mathcal{V}_{3}, \mathcal{V}_{4}\right) \triangleq \mathbf{g}\left(\mathcal{V}_{1}\right)+\mathbf{g}\left(\mathcal{V}_{2}\right)+\mathbf{g}\left(\mathcal{V}_{3} \mathcal{V}_{4}\right)+\mathbf{g}\left(\mathcal{V}_{1} \mathcal{V}_{2} \mathcal{V}_{3}\right)+\mathbf{g}\left(\mathcal{V}_{1} \mathcal{V}_{2} \mathcal{V}_{4}\right)
\end{aligned}
$$

With these definitions, the Ingleton inequality (9) is written

$$
J_{\mathrm{g}}^{+}\left(\mathcal{V}_{1}, \mathcal{V}_{2}, \mathcal{V}_{3}, \mathcal{V}_{4}\right) \geq J_{\mathrm{g}}^{-}\left(\mathcal{V}_{1}, \mathcal{V}_{2}, \mathcal{V}_{3}, \mathcal{V}_{4}\right)
$$

It is straightforward to prove that $(\mathcal{Y}, \mathbf{g})$ is a polymatroid. We must additionally show that it is Ingletonian. Let

$$
U=\{(1,2),(1,3),(2,3),(1,4),(2,4)\} .
$$

be the collection of $\alpha \subseteq \mathcal{N}_{4}$ such that the summand $\mathrm{g}\left(\mathcal{V}_{i}, i \in \alpha\right)$ appears in $J_{\mathrm{g}}^{+}\left(\mathcal{V}_{1}, \mathcal{V}_{2}, \mathcal{V}_{3}, \mathcal{V}_{4}\right)$. Let $Q \triangleq\left\{\alpha \subseteq \mathcal{N}_{4}: \mathbf{h}(\mathcal{Y})-\epsilon \leq \mathbf{h}\left(\mathcal{V}_{i}, i \in \alpha\right)\right\}$. Thus $Q$ identifies summands in $J^{+}$and $J^{-}$for which the $\epsilon$-perturbation in (8) bites. Note that if $\alpha \in Q$, then $\mathbf{g}\left(\mathcal{V}_{i}, i \in \alpha\right) \geq \mathbf{g}\left(\mathcal{V}_{i}, i \in \beta\right)$ for all $\beta \subseteq \mathcal{N}_{4}$.

We will now proceed on a case-by-case basis, proving that (9) holds in the following distinct and exhaustive cases.

Case 1: $Q \cap U=\emptyset$.

Inequality (9) clearly holds and follows from the fact that $(\mathcal{Y}, \mathbf{h})$ is Ingletonian, $J_{\mathbf{g}}^{+}=J_{\mathbf{h}}^{+}$and $J_{\mathrm{g}}^{-} \leq J_{\mathbf{h}}^{-}$.

Case 2: $Q \cap U=\{(1,2)\}$.

In this case, $\mathbf{h}\left(\mathcal{V}_{1} \mathcal{V}_{2}\right) \geq \mathbf{h}(\mathcal{Y})-\epsilon$. By monotonicity of polymatroids $(\mathrm{R} 2), \mathbf{h}\left(\mathcal{V}_{1} \mathcal{V}_{2} \mathcal{V}_{3}\right) \geq$ $\mathbf{h}(\mathcal{Y})-\epsilon$. The left hand side of (10) thus becomes

$$
J_{\mathrm{g}}^{+}\left(\mathcal{V}_{1}, \mathcal{V}_{2}, \mathcal{V}_{3}, \mathcal{V}_{4}\right)=J_{\mathbf{h}}^{+}\left(\mathcal{V}_{1}, \mathcal{V}_{2}, \mathcal{V}_{3}, \mathcal{V}_{4}\right)-\mathbf{h}\left(\mathcal{V}_{1} \mathcal{V}_{2}\right)+\mathbf{h}(\mathcal{Y})-\epsilon
$$

Similarly, its right hand side can be shown to be bounded above by

$$
J_{\mathrm{g}}^{-}\left(\mathcal{V}_{1}, \mathcal{V}_{2}, \mathcal{V}_{3}, \mathcal{V}_{4}\right)=J_{\mathbf{h}}^{-}\left(\mathcal{V}_{1}, \mathcal{V}_{2}, \mathcal{V}_{3}, \mathcal{V}_{4}\right)-\mathbf{h}\left(\mathcal{V}_{1} \mathcal{V}_{2} \mathcal{V}_{3}\right)+\mathbf{h}(\mathcal{Y})-\epsilon
$$


Thus (9) holds, since $(\mathcal{Y}, \mathbf{h})$ is Ingletonian and $\mathbf{h}\left(\mathcal{V}_{1} \mathcal{V}_{2}\right) \leq \mathbf{h}\left(\mathcal{V}_{1} \mathcal{V}_{2} \mathcal{V}_{3}\right)$. A similar approach may be used when $Q \cap U$ is any one of $(1,3),(1,4),(2,3)$ or $(2,4)$.

Case 3: $Q \cap U=\{(1,3),(2,3)\}$.

As $\mathrm{g}$ is a polymatroid, $\mathrm{g}\left(\mathcal{V}_{1} \mathcal{V}_{2}\right)+\mathrm{g}\left(\mathcal{V}_{1} \mathcal{V}_{4}\right)+\mathrm{g}\left(\mathcal{V}_{2} \mathcal{V}_{4}\right) \geq \mathrm{g}\left(\mathcal{V}_{1}\right)+\mathrm{g}\left(\mathcal{V}_{2}\right)+\mathrm{g}\left(\mathcal{V}_{1} \mathcal{V}_{2} \mathcal{V}_{4}\right)$ and hence (9) holds as $\mathbf{g}\left(\mathcal{V}_{1} \mathcal{V}_{3}\right)+\mathbf{g}\left(\mathcal{V}_{2} \mathcal{V}_{3}\right)=2 \mathrm{~g}(\mathcal{Y}) \geq \mathbf{g}\left(\mathcal{V}_{3} \mathcal{V}_{4}\right)+\mathbf{g}\left(\mathcal{V}_{1} \mathcal{V}_{2} \mathcal{V}_{3}\right)$. Similarly, (9) holds if $Q \cap U=\{(1,4),(2,4)\}$.

Case 4: $Q \cap U=\{(1,2),(1,3)\}$.

Again, $\mathbf{g}\left(\mathcal{V}_{2} \mathcal{V}_{3}\right)+\mathbf{g}\left(\mathcal{V}_{1} \mathcal{V}_{4}\right)+\mathbf{g}\left(\mathcal{V}_{2} \mathcal{V}_{4}\right) \geq \mathbf{g}\left(\mathcal{V}_{1}\right)+\mathbf{g}\left(\mathcal{V}_{2}\right)+\mathbf{g}\left(\mathcal{V}_{3} \mathcal{V}_{4}\right)$ and consequently (9) holds because $\mathrm{g}$ is a polymatroid. Using the same argument, (9) also holds when $Q \cap U$ is either $\{(1,2),(1,4)\},\{(1,2),(2,3)\}$ or $\{(1,2),(2,4)\}$.

Case 5: $Q \cap U=\{(1,3),(2,4)\}$.

Now $\mathbf{g}$ is a polymatroid and hence $\mathbf{g}\left(\mathcal{V}_{1} \mathcal{V}_{2}\right)+\mathbf{g}\left(\mathcal{V}_{2} \mathcal{V}_{3}\right)+\mathbf{g}\left(\mathcal{V}_{1} \mathcal{V}_{4}\right) \geq \mathbf{g}\left(\mathcal{V}_{1}\right)+\mathbf{g}\left(\mathcal{V}_{2}\right)+\mathbf{g}\left(\mathcal{V}_{1} \mathcal{V}_{2} \mathcal{V}_{3}\right)$ Consequently, (9) holds as in the previous case. Similarly, (9) holds when $Q \cap U=\{(1,4),(2,3)\}$.

Case 6: $Q \cap U=\{(1,3),(1,4)\}$.

In this case, (9) holds because $\mathbf{g}\left(\mathcal{V}_{1} \mathcal{V}_{2}\right)+\mathbf{g}\left(\mathcal{V}_{2} \mathcal{V}_{3}\right)+\mathbf{g}\left(\mathcal{V}_{2} \mathcal{V}_{4}\right) \geq \mathbf{g}\left(\mathcal{V}_{1}\right)+\mathbf{g}\left(\mathcal{V}_{2}\right)+\mathbf{g}\left(\mathcal{V}_{2} \mathcal{V}_{3} \mathcal{V}_{4}\right)$

Case 7: $|Q \cap U|=3$ and $Q \cap U=\{(1,2),(2,3),(2,4)\}$.

Inequality (9) follows from

$$
\begin{aligned}
\mathbf{g}\left(\mathcal{V}_{1} \mathcal{V}_{3}\right)+\mathbf{g}\left(\mathcal{V}_{1} \mathcal{V}_{4}\right) & \geq \mathbf{g}\left(\mathcal{V}_{1}\right)+\mathbf{g}\left(\mathcal{V}_{1} \mathcal{V}_{3} \mathcal{V}_{4}\right) \\
& \geq \mathbf{g}\left(\mathcal{V}_{1}\right)+\mathbf{g}\left(\mathcal{V}_{3} \mathcal{V}_{4}\right)
\end{aligned}
$$

A similar approach can be used for other cases when $|Q \cap U| \geq 3$.

In Theorem 1, we proved that the $\epsilon$-perturbation of an Ingletonian polymatroid is also Ingletonian. Theorem 2 shows that $\epsilon$-perturbation also preserves representability.

Theorem 2: Suppose $(\mathcal{X}, \mathbf{h})$ is representable and let $\left(\mathcal{X}, \mathbf{g}_{\epsilon}\right)$ be its $\epsilon$-perturbation (8). Then $\left(\mathcal{X}, \mathbf{g}_{\epsilon}\right)$ is almost representable for any $0 \leq \epsilon \leq \mathbf{h}(\mathcal{X})$. It is also representable if $\epsilon \in \mathbb{Z}$.

Before we prove Theorem 2 , we require some basic results regarding vector subspaces. Let $A$ be a vector subspace of $W$. Define $A^{*}$ as a subspace of $W$ such that 
1) $\left\langle A, A^{*}\right\rangle=W$, and hence $H\left(A, A^{*}\right)=H(W)$

2) $A \cap A^{*}=\{\mathbf{0}\}$.

Clearly, any vector $\mathbf{u} \in \mathbf{W}$ can be written uniquely as $\mathbf{u}=\mathbf{u}_{1}+\mathbf{u}_{2}$ where $\mathbf{u}_{1} \in A^{*}$ and $\mathbf{u}_{2} \in A$. We will refer to $\mathbf{u}_{1} \triangleq T_{A}(\mathbf{u})$ as the projection of $\mathbf{u}$ away from $A$. While $T_{A}(\mathbf{u})$ depends on the choice of $A^{*}$, it can be directly verified that the following lemma holds for all legitimate choices of $A^{*}$.

Lemma 2: Let $B$ and $C$ be subspaces of $W$. Then for any subspace $A$ of $W$,

$$
T_{A}(B) \triangleq\left\{T_{A}(\mathbf{u}): \mathbf{u} \in B\right\}
$$

is a subspace of $W$. Furthermore,

$$
\begin{aligned}
H\left(T_{A}(B)\right) & =H(B \mid A), \\
H\left(T_{A}\left(\left\langle B_{j}, j \in \beta\right\rangle\right)\right) & =H\left(T_{A}\left(B_{j}\right), j \in \beta\right) .
\end{aligned}
$$

Consequently,

1) $H\left(B_{j}, j \in \beta\right) \geq H\left(T_{A}\left(B_{j}\right), j \in \beta\right) \geq H\left(B_{j}, j \in \beta\right)-H(A)$

2) If $H(B \mid C)=0$ (i.e. $B \subseteq C)$, then $H\left(T_{A}(B) \mid T_{A}(C)\right)=0$. More generally, if $H\left(B \mid C_{i}, i \in \alpha\right)=$ 0 , then $H\left(T_{A}(B) \mid T_{A}\left(C_{i}\right), i \in \alpha\right)=0$.

3) If $B \cap A=\{0\}$ (i.e. $I(A ; B)=0$ ), then $H(B)=H\left(T_{A}(B)\right)$.

The projection operator $T_{A}(\cdot)$ has a natural interpretation. Specifically, let $\left\{X_{1}, \ldots, X_{n}, A\right\}$ be a collection of subspaces in $W$, which induces a representable polymatroid in the usual way. By Lemma 2, there exists subspaces $\left\{B_{1}, \ldots, B_{n}\right\}$ of $W$ such that $B_{i} \triangleq T_{A}\left(X_{i}\right)$ and

$$
H\left(B_{i}, i \in \alpha\right)=H\left(X_{i}, i \in \alpha \mid A\right) .
$$

In other words, using the projection operator $T_{A}(\cdot)$, one can transform any set of subspaces $\left\{X_{1}, \ldots, X_{n}\right\}$ into another set $\left\{B_{1}, \ldots, B_{n}\right\}$ such that $H\left(B_{i}, i \in \alpha\right)=H\left(X_{i}, i \in \alpha \mid A\right) . \quad$ Proof: [Proof of Theorem 2] Suppose $(\mathcal{X}, \mathbf{h})$ is representable. If $\mathbf{h}(\mathcal{X})=0$, then the theorem is trivial. Now suppose $\mathbf{h}(\mathcal{X})>0$ and hence $\mathbf{h}(\mathcal{X}) \geq 1$ (since it is the dimension of a space). We begin by proving that $\left(\mathcal{X}, \mathbf{g}_{\epsilon}\right)$ is representable (and hence almost representable) when $\epsilon=1$. The argument will subsequently be extended to cover other values of $\epsilon$. 
First, as $(\mathcal{X}, \mathbf{h})$ is representable, there exists subspaces $V_{i}, i \in \mathcal{N}$ such that

$$
\mathbf{h}\left(X_{i}, i \in \alpha\right)=H\left(V_{i}, i \in \alpha\right) \triangleq \operatorname{dim}\left\langle V_{i}, i \in \alpha\right\rangle .
$$

Assume without loss of generality that these subspaces are over an underlying field $\mathbb{F}_{q}$. For any positive integer $m, \mathbb{F}_{q}$ can be regarded as a subfield of $\mathbb{F}_{q^{m}}$. In fact, for each $i$, let $V_{i}^{(m)}$ be the subspaces over $\mathbb{F}_{q^{m}}$ spanned by $V_{i}$. Then

$$
\operatorname{dim}\left\langle V_{i}^{(m)}, i \in \alpha\right\rangle=\operatorname{dim}\left\langle V_{i}, i \in \alpha\right\rangle
$$

or equivalently, $H\left(V_{i}^{(m)}, i \in \alpha\right)=H\left(V_{i}, i \in \alpha\right)$.

Let $C=H\left(V_{\mathcal{N}}\right)$ and $Q \triangleq\left\{\alpha \subseteq \mathcal{N}: H\left(V_{\alpha}\right)<C\right\}$. Then for each $\alpha \in Q$,

$$
H\left(V_{\alpha}^{(m)}\right)=H\left(V_{\alpha}\right) \leq C-1 .
$$

The volume (i.e. cardinality) of $\left\langle V_{i}^{(m)}, i \in \alpha\right\rangle$ is at most $\left(q^{m}\right)^{C-1}$ while the volume of $\left\langle V_{i}^{(m)}, i \in\right.$ $\mathcal{N}\rangle$ is $\left(q^{m}\right)^{C}$. Hence, for sufficiently large $m, \bigcup_{\alpha \in Q} V_{i}^{(m)}$ is a proper subset of $\left\langle V_{i}^{(m)}, i \in \mathcal{N}\right\rangle$.

Let $\mathbf{u} \in\left\langle V_{i}^{(m)}, i \in \mathcal{N}\right\rangle$ but not in $\bigcup_{\alpha \in Q} V_{i}^{(m)}$. Let $A=\langle\mathbf{u}\rangle$ and define

$$
B_{i} \triangleq T_{A}\left(V_{i}^{(m)}\right) \text {. }
$$

By Lemma2, it is straightforward to prove that $\mathbf{g}_{\epsilon}(\alpha)=H\left(B_{i}, i \in \alpha\right)$. Hence, $\mathbf{g}_{\epsilon}$ is representable. Repeating the same argument multiple times, we can also prove that the theorem also holds when $\epsilon$ is a positive integer.

Now, suppose $\epsilon=k / \ell$ is rational. For any representable $(\mathcal{X}, \mathbf{h})$, it is easy to find another representable $(\mathcal{X}, \mathbf{f})$ such that $\mathbf{f}=\ell \mathbf{h}$. Consequently

$$
\begin{aligned}
\mathbf{g}_{\epsilon}(\mathcal{A}) & =\min (\mathbf{h}(\mathcal{A}), \mathbf{h}(\mathcal{X})-k / \ell) \\
& =\frac{1}{\ell} \min (\ell \mathbf{h}(\mathcal{A}), \ell \mathbf{h}(\mathcal{X})-k) \\
& =\frac{1}{\ell} \min (\mathbf{f}(\mathcal{A}), \mathbf{f}(\mathcal{X})-k) .
\end{aligned}
$$

is almost representable.

Finally, the remaining case when $\epsilon$ is irrational can be proved by a continuity argument. Specifically, let $\nu_{j}$ be a sequence of rational numbers converging to $\epsilon$, then it is easy to prove that $\lim _{j \rightarrow \infty} \mathbf{g}_{\nu_{j}}=\mathbf{g}_{\epsilon}$. Hence, $\mathbf{g}_{\epsilon}$ is almost representable. 
The final result of this Section, Theorem 3 is a direct consequence of the following proposition.

Proposition 2: Let $\left\{\left(\mathcal{X}, \mathbf{h}_{i}\right)\right\}_{i=1}^{\infty}$ be a sequence of representable polymatroids such that $\lim _{i \rightarrow \infty} c_{i} \mathbf{h}_{i}=$ $\mathbf{h}_{0}$ for some positive sequence of numbers $\left\{c_{i}\right\}_{i=1}^{\infty}$. Suppose $0 \leq \epsilon \leq \mathbf{h}_{0}(\mathcal{X})$. For each $i$, define

$$
\begin{aligned}
\mathbf{g}_{i}(\mathcal{A}) & \triangleq \min \left(c_{i} \mathbf{h}_{i}(\mathcal{A}), c_{i} \mathbf{h}_{i}(\mathcal{X})-\epsilon\right) \\
\mathbf{g}_{0}(\mathcal{A}) & \triangleq \min \left(\mathbf{h}_{0}(\mathcal{A}), \mathbf{h}_{0}(\mathcal{X})-\epsilon\right)
\end{aligned}
$$

Then $\lim _{i \rightarrow \infty} \mathbf{g}_{i}=\mathbf{g}_{0}$ and $\mathbf{g}_{0}$ is almost representable.

Proof: If $\epsilon=\mathbf{h}_{0}(\mathcal{X})$, then the proposition is obvious. Now, suppose $\epsilon<\mathbf{h}_{0}(\mathcal{X})$. By the continuity of $\min (a, b)$,

$$
\lim _{i \rightarrow \infty}\left(\min \left(c_{i} \mathbf{h}_{i}(\mathcal{A}), c_{i} \mathbf{h}_{i}(\mathcal{X})-\epsilon\right)\right)=\min \left(\lim _{i \rightarrow \infty} c_{i} \mathbf{h}_{i}(\mathcal{A}), \lim _{i \rightarrow \infty} c_{i} \mathbf{h}_{i}(\mathcal{X})-\epsilon\right)
$$

and hence $\lim _{i \rightarrow \infty} \mathbf{g}_{i}=\mathbf{g}_{0}$.

On the other hand, since $\lim _{i \rightarrow \infty} c_{i} \mathbf{h}_{i}=\mathbf{h}_{0}$ and $0 \leq \epsilon<\mathbf{h}_{0}(\mathcal{X})$, we have for sufficiently large $i$ that $0 \leq \epsilon \leq c_{i} \mathbf{h}_{i}(\mathcal{X})$ or equivalently $0 \leq \epsilon / c_{i} \leq \mathbf{h}_{i}(\mathcal{X})$. As $\mathbf{h}_{i}$ is representable, $\mathbf{g}_{i} / c_{i}$ and hence $\mathbf{g}_{i}$ are almost representable by Theorem 2 , Consequently, its limit $\mathbf{g}_{0}$ is also almost representable.

Theorem 3: Suppose $(\mathcal{X}, \mathbf{h})$ is almost representable. Let $(\mathcal{X}, \mathbf{g})$ be its $\epsilon$-perturbed polymatroid as defined in (8). Then $\mathrm{g}$ is almost representable.

\section{MAIN RESUlTS}

The main result of this paper is the following theorem, which is a direct consequence of Theorem 5, which we prove in this section, and Theorem 6 in the following section (which establishes the existence of certain matroids required for Theorem (5).

Theorem 4 (Insufficiency of Ingleton's inequalities): There exists an Ingletonian polymatroid that is not cc-representable. Consequently, there are linear inequalities satisfied by all representable polymatroids but not implied by Ingleton's inequalities.

So far, we have defined the concept of $\epsilon$-perturbation and proved that it preserves both the Ingletonian property and representability. We will now use $\epsilon$-perturbation to construct an 
Ingletonian polymatroid that is not cc-representable. First, we will need to establish a key lemma concerning connected matroids [13, Chapter 4].

Definition 3 (Connected Matroid): A matroid $(\mathcal{X}, \mathbf{h})$ is connected if for any pair of $X_{1}$ and $X_{2}$ in $\mathcal{X}$, there exists a circuit contains both $X_{1}$ and $X_{2}$.

Definition 4: Let $M=(\mathcal{X}, \mathbf{h})$ be a matroid. Define $\mathcal{I}(M)$ as the set of equalities of the forms $H(\mathcal{A} \mid \mathcal{B})=0$ or $I(\mathcal{A} ; \mathcal{B})=0$ satisfied by $M$.

Lemma 3: If a matroid $M=(\mathcal{X}, \mathbf{h})$ is connected and $(\mathcal{X}, \mathbf{g})$ is any polymatroid that satisfies the set of $\mathcal{I}(M)$ equalities induced by $M$, then $\mathbf{g}\left(X_{i}\right)$ is constant for all $X_{i} \in \mathcal{X}$ and $\mathbf{g}=c \mathbf{h}$ for some $c \geq 0$.

Proof: Suppose $X_{1}, X_{2}$ belong to a circuit in the matroid $M$. Then there exists a subset of variables $\mathcal{A}$ such that

$$
H\left(X_{1} \mid X_{2}, \mathcal{A}\right)=H\left(X_{2} \mid X_{1}, \mathcal{A}\right)=I\left(X_{1} ; \mathcal{A}\right)=I\left(X_{2} ; \mathcal{A}\right)=0
$$

where (22) is with respect to $\mathbf{h}$. By assumption, $\mathbf{g}$ satisfies $\mathcal{I}(M)$, which includes (22). It is easy to prove that if $(\mathcal{X}, \mathbf{g})$ is a polymatroid also satisfying (22), then $\mathbf{g}\left(X_{1}\right)=\mathbf{g}\left(X_{2}\right)$. By the connectedness of $M, \mathbf{g}\left(X_{i}\right)$ is constant for all $X_{i} \in \mathcal{X}$.

Now, let $\mathcal{B}$ be any subset of $\mathcal{X}$. Since $(\mathcal{X}, \mathbf{h})$ is a matroid, there exists $\mathcal{A} \subset \mathcal{B}$ such that $\mathbf{h}(\mathcal{B} \mid \mathcal{A})=0$ and $\mathbf{h}(\mathcal{A})=\sum_{X_{i} \in \mathcal{A}} \mathbf{h}\left(X_{i}\right)$, and these identities belong to $\mathcal{I}(M)$. By assumption, $(\mathcal{X}, \mathbf{g})$ also satisfies $\mathcal{I}(M)$, and hence $\mathbf{g}(\mathcal{B} \mid \mathcal{A})=0$ and $\mathbf{g}(\mathcal{A})=\sum_{X_{i} \in \mathcal{A}} \mathbf{g}\left(X_{i}\right)$. Thus $\mathbf{h}(\mathcal{B})=$ $|\mathcal{A}| \mathbf{h}\left(X_{i}\right)$ and $\mathbf{g}(\mathcal{B})=|\mathcal{A}| \mathbf{g}\left(X_{i}\right)$.

The main result of this paper hinges on the following theorem. It describes an approach to adhere two representable matroids together in such a way that the resulting polymatroid is not cc-representable. We establish this theorem for connected matroids that are even (and odd) representable but not almost odd (even) representable. The existence of such strictly even and strictly odd matroids will be established in Theorem 6. Together with Theorem 5, this provides the proof of Theorem 4 , namely the insufficiency of the Ingleton inequalities .

Theorem 5: Let $\left(\mathcal{X}_{1}, \Phi_{1}\right)$ (and $\left(\mathcal{X}_{2}, \Phi_{2}\right)$ ) be an even (and odd) connected representable matroid that is not almost odd (almost even) representable. Let $(\mathcal{X}, \Phi)$ be the direct sum of these two matroids, namely $\mathcal{X}=\mathcal{X}_{1} \cup \mathcal{X}_{2}$ and $\Phi(\mathcal{A})=\Phi_{1}\left(\mathcal{A} \cap \mathcal{X}_{1}\right)+\Phi_{2}\left(\mathcal{A} \cap \mathcal{X}_{2}\right)$ for all $\mathcal{A} \subseteq \mathcal{X}$. Suppose $0<\epsilon \leq \min \left(\Phi_{1}\left(\mathcal{X}_{1}\right), \Phi_{2}\left(\mathcal{X}_{2}\right)\right)$. Then $\left(\mathcal{X}, \Phi^{\epsilon}\right)$ is not cc-representable. 
Proof: By definition of $\epsilon$-perturbation (8), it is easily verified that

$$
\begin{array}{ll}
\Phi^{\epsilon}(\mathcal{A})=\Phi_{1}(\mathcal{A}), & \forall \mathcal{A} \subseteq \mathcal{X}_{1} \\
\Phi^{\epsilon}(\mathcal{B}) & =\Phi_{2}(\mathcal{B}), \quad \forall \mathcal{B} \subseteq \mathcal{X}_{2}
\end{array}
$$

Hence, $\Phi^{\epsilon}$ satisfies all the equalities $\mathcal{I}\left(M_{1}\right)$ and $\mathcal{I}\left(M_{2}\right)$.

Suppose to the contrary that $\Phi^{\epsilon} \in \overline{\operatorname{con}}(\Upsilon[\mathcal{X}])$. Then by definition there exists a sequence of points $\mathbf{h}_{i} \in \operatorname{con}(\Upsilon[\mathcal{X}])$ such that $\lim _{i \rightarrow \infty} \mathbf{h}_{i}=\Phi^{\epsilon}$. As each $\mathbf{h}_{i}$ is a point in a $2^{|\mathcal{X}|}$-dimensional Euclidean space, Caratheodory's theorem allows each $\mathbf{h}_{i}$ to be written

$$
\mathbf{h}_{i}=\sum_{j=1}^{2^{|\mathcal{X}|}+1} c_{i, j} \mathbf{f}_{i, j}
$$

where $c_{i, j} \geq 0$ and $d_{i, j} \mathbf{f}_{i, j}$ is representable for some $d_{i, j}>0$.

Assume without loss of generality that $\mathbf{h}_{i}(\mathcal{X})=\mathbf{f}_{i, j}(\mathcal{X})=\Phi^{\epsilon}(\mathcal{X})$ for all $i, j$. Then all $\mathbf{f}_{i, j}$ are contained in the compact set $\left\{\mathbf{f} \in \mathbb{R}^{2^{|\mathcal{X}|}}: 0 \leq \mathbf{f}(\mathcal{A}) \leq \Phi^{\epsilon}(\mathcal{X})\right.$ for all $\left.\mathcal{A} \subseteq \mathcal{X}\right\}$ and $\sum_{j=1}^{2^{|\mathcal{X}|}+1} c_{i, j}=$ 1 (and hence $0 \leq c_{i, j} \leq 1$ ). According to the Bolzano-Weierstrass theorem, any bounded sequence in a finite dimensional Euclidean space has a convergent subsequence. We may therefore assume without loss of generality the existence of the following limits for any $j=1, \ldots, 2^{|\mathcal{X}|}+1$

$$
\begin{aligned}
& \lim _{i \rightarrow \infty} c_{i, j}=c_{j} \\
& \lim _{i \rightarrow \infty} \mathbf{f}_{i, j}=\mathbf{f}_{j} .
\end{aligned}
$$

Hence $\Phi^{\epsilon}=\sum_{j=1}^{2^{|\mathcal{X}|}+1} c_{j} \mathbf{f}_{j}$.

As each $\mathbf{f}_{j}$ is the limit of a sequence of polymatroids $\left\{\mathbf{f}_{i, j}\right\}_{i=1}^{\infty},\left(\mathcal{X}, \mathbf{f}_{j}\right)$ is also a polymatroid for all $j$. Therefore, $\left(\mathcal{X}, \mathbf{f}_{j}\right)$ also satisfies equalities $\mathcal{I}\left(M_{1}\right)$ and $\mathcal{I}\left(M_{2}\right)$. On the other hand, as $\epsilon>0, \Phi^{\epsilon}\left(\mathcal{X}_{1}\right)+\Phi^{\epsilon}\left(\mathcal{X}_{2}\right)>\Phi^{\epsilon}(\mathcal{X})$, there is at least one $j$ such that

$$
\mathbf{f}_{j}\left(\mathcal{X}_{1}\right)+\mathbf{f}_{j}\left(\mathcal{X}_{2}\right)>\mathbf{f}_{j}(\mathcal{X})
$$

Consequently, both $\mathbf{f}_{j}\left(\mathcal{X}_{1}\right)$ and $\mathbf{f}_{j}\left(\mathcal{X}_{2}\right)$ are positive as $\mathbf{f}_{j}$ is a polymatroid. Furthermore, since $\left(\mathcal{X}_{1}, \Phi_{1}\right)$ and $\left(\mathcal{X}_{2}, \Phi_{2}\right)$ are connected matroids, by Lemma 3 , there exists positive constants $c$ and $c^{\prime}$ such that

$$
\begin{aligned}
\mathbf{f}_{j}(\mathcal{A})=c \Phi_{1}(\mathcal{A}), & \forall \mathcal{A} \subseteq \mathcal{X}_{1} \\
\mathbf{f}_{j}(\mathcal{B})=c^{\prime} \Phi_{2}(\mathcal{B}), & \forall \mathcal{B} \subseteq \mathcal{X}_{2}
\end{aligned}
$$


So far, we have proved that if $\Phi^{\epsilon} \in \overline{\operatorname{con}}(\Upsilon[\mathcal{X}])$, then there exists a sequence of polymatroids $\left\{\left(\mathcal{X}, \mathbf{f}_{i, j}\right)\right\}_{i=1}^{\infty}$ such that (1) $d_{i, j} \mathbf{f}_{i, j}$ is representable for some $d_{i, j}>0$, and (2) its limit $\mathbf{f}_{j}$ satisfies (26) and (27). We may further assume without loss of generality that $d_{i, j} \mathbf{f}_{i, j}$ is either even representable or odd representable for all $i$. Suppose first that all $d_{i, j} \mathbf{f}_{i, j}$ are even representable for all $i$. The fact that $\lim _{i \rightarrow \infty} \mathbf{f}_{i, j}(\mathcal{B})=\mathbf{f}_{j}(\mathcal{B})=c^{\prime} \Phi_{2}(\mathcal{B})$ thus implies that $\left(\mathcal{X}, \Phi_{2}\right)$ is almost even representable, contradicting the hypothesis. Similarly, contradiction occurs if $d_{i, j} \mathbf{f}_{i, j}$ are odd representable for all $i$. Contradiction occurs in both cases and hence the theorem is proved.

\section{Strictly Odd AND EVEn Matroids}

In this section, we will construct two representable matroids $\left(\mathcal{X}_{1}, \Phi_{1}\right)$ and $\left(\mathcal{X}_{2}, \Phi_{2}\right)$ that satisfy the conditions given in Theorem 5 , These matroids correspond to the first and second networks in $[15$, Section II].

Define the matroid $\left(\mathcal{X}_{1}, \Phi_{1}\right)$ with $\Phi_{1}(\cdot)=\operatorname{dim}\langle\cdot\rangle$ and ground set

$$
\begin{aligned}
\mathcal{X}_{1} & \triangleq\left\{Y_{1}, Y_{2}, Y_{3}, W_{1}, W_{2}, W_{3}, W_{4}\right\} \\
Y_{i} & =\left\langle\mathbf{u}_{i}\right\rangle, i=1,2,3 \\
W_{1} & =\left\langle\mathbf{u}_{1}+\mathbf{u}_{2}\right\rangle \\
W_{2} & =\left\langle\mathbf{u}_{2}+\mathbf{u}_{3}\right\rangle \\
W_{3} & =\left\langle\mathbf{u}_{1}+\mathbf{u}_{2}+\mathbf{u}_{3}\right\rangle \\
W_{4} & =\left\langle\mathbf{u}_{1}+\mathbf{u}_{3}\right\rangle .
\end{aligned}
$$

where $\mathbf{u}_{1}, \mathbf{u}_{2}, \mathbf{u}_{3}$ are linearly independent vectors over a finite field of even characteristic. Clearly $\left(\mathcal{X}_{1}, \Phi_{1}\right)$ is even representable (and hence Ingletonian). In fact, this is the Fano matroid, $F_{7}$. 
Define the second matroid $\left(\mathcal{X}_{2}, \Phi_{2}\right)$ with $\Phi_{2}(\cdot)=\operatorname{dim}\langle\cdot\rangle$ and

$$
\begin{aligned}
\mathcal{X}_{2} & \triangleq\left\{Z_{1}, \ldots, Z_{5}, V_{1}, \ldots, V_{8}\right\}, \\
Z_{i} & =\left\langle\mathbf{u}_{i}\right\rangle, i=1, \ldots, 5 \\
V_{1} & =\left\langle\mathbf{u}_{1}+\mathbf{u}_{2}+\mathbf{u}_{3}\right\rangle \\
V_{2} & =\left\langle\mathbf{u}_{3}+\mathbf{u}_{4}+\mathbf{u}_{5}\right\rangle \\
V_{3} & =\left\langle\mathbf{u}_{1}+\mathbf{u}_{2}\right\rangle \\
V_{4} & =\left\langle\mathbf{u}_{1}+\mathbf{u}_{3}\right\rangle \\
V_{5} & =\left\langle\mathbf{u}_{2}+\mathbf{u}_{3}\right\rangle \\
V_{6} & =\left\langle\mathbf{u}_{3}+\mathbf{u}_{4}\right\rangle \\
V_{7} & =\left\langle\mathbf{u}_{3}+\mathbf{u}_{5}\right\rangle \\
V_{8} & =\left\langle\mathbf{u}_{4}+\mathbf{u}_{5}\right\rangle
\end{aligned}
$$

where $\left\{\mathbf{u}_{1}, \ldots \mathbf{u}_{5}\right\}$ are linearly independent over a finite field of odd characteristic. Clearly $\left(\mathcal{X}_{1}, \Phi_{1}\right)$ is odd representable (and hence Ingletonian).

It is easy to prove that $\left(\mathcal{X}_{1}, \Phi_{1}\right)$ and $\left(\mathcal{X}_{2}, \Phi_{2}\right)$ are both connected. Furthermore, $\left(\mathcal{X}_{1}, \Phi_{1}\right)$ satisfies the following equalities (recalling the notational conventions (2), (3))

$$
\begin{array}{rlrl}
H\left(Y_{1}, Y_{2}, Y_{3}\right)=\sum_{i=1}^{3} H\left(Y_{i}\right) & \\
H\left(W_{1} \mid Y_{1} Y_{2}\right) & =0 & H\left(W_{2} \mid Y_{2} Y_{3}\right) & =0 \\
H\left(W_{3} \mid Y_{1} W_{2}\right) & =0 & H\left(W_{4} \mid W_{1} W_{2}\right) & =0 \\
H\left(Y_{1} \mid Y_{3} W_{4}\right) & =0 & H\left(Y_{2} \mid W_{3} W_{4}\right) & =0 \\
H\left(Y_{3} \mid W_{1} W_{3}\right) & =0 &
\end{array}
$$

It is worth pointing out explicitly that $H\left(W_{4} \mid W_{1} W_{2}\right)=0$ because all vectors are defined over a finite field of even characteristic (hence, $\mathbf{u}_{1}+\mathbf{u}_{3}=\mathbf{u}_{1}+\mathbf{u}_{2}+\mathbf{u}_{2}+\mathbf{u}_{3}$ ).

Simiarly, $\left(\mathcal{X}_{2}, \Phi_{2}\right)$ satisfies

$$
H\left(Z_{1}, \ldots, Z_{5}\right)=\sum_{i=1}^{5} H\left(Z_{i}\right)
$$




$$
\begin{array}{rlrl}
H\left(V_{1} \mid Z_{1} Z_{2} Z_{3}\right) & =0 & H\left(V_{2} \mid Z_{3} Z_{4} Z_{5}\right) & =0 \\
H\left(V_{3} \mid Z_{1} Z_{2}\right) & =0 & H\left(V_{4} \mid Z_{1} Z_{3}\right) & =0 \\
H\left(V_{5} \mid Z_{2} Z_{3}\right) & =0 & H\left(V_{6} \mid Z_{3} Z_{4}\right) & =0 \\
H\left(V_{7} \mid Z_{3} Z_{5}\right) & =0 & H\left(V_{8} \mid Z_{4} Z_{5}\right) & =0 \\
H\left(Z_{1} \mid V_{1} V_{5}\right) & =0 & H\left(Z_{2} \mid V_{1} V_{4}\right) & =0 \\
H\left(Z_{3} \mid V_{1} V_{3}\right) & =0 & H\left(Z_{3} \mid V_{3}, \ldots, V_{8}\right) & =0 \\
H\left(Z_{3} \mid V_{2} V_{8}\right) & =0 & H\left(Z_{4} \mid V_{2} V_{7}\right) & =0 \\
H\left(Z_{5} \mid V_{2} V_{6}\right) & =0 . &
\end{array}
$$

In this case, we emphasis that $H\left(Z_{3} \mid V_{3}, \ldots, V_{8}\right)=0$ holds because the characteristic of the underlying field is odd.

Theorem 6: $\left(\mathcal{X}, \Phi_{1}\right)$ defined by (28) is even representable but not almost odd representable. Simiarly, $\left(\mathcal{X}, \Phi_{2}\right)$ defined by (34) is odd representable but not almost even representable.

Before we prove Theorem 6, we will need two lemmas, which provide some elementary results from linear algebra.

Lemma 4: Let $\left\{B_{1}, \ldots, B_{n}\right\}$ and $C$ be subspaces of $W$. Then for any $\alpha \subseteq \mathcal{N}$, there exists a subspace $A$ such that $H(A)=H\left(C \mid B_{j}, j \in \alpha\right)$ and $H\left(T_{A}(C) \mid T_{A}\left(B_{j}\right), j \in \alpha\right)=0$. Furthermore, for a given sequence of subspaces $\left\{B_{1}^{i}, \ldots, B_{n}^{i}, C^{i}\right\}_{i=1}^{\infty}$ and $k(i)>0$ such that

$$
\lim _{i \rightarrow \infty} \frac{1}{k(i)} H\left(C^{i} \mid B_{j}^{i}, j \in \alpha\right)=0,
$$

there exists a sequence of subspaces $\left\{A^{i}\right\}_{i=1}^{\infty}$ such that for all $\beta \subseteq \mathcal{N}_{n}$

$$
\begin{aligned}
H\left(A^{i}\right) & =H\left(C^{i} \mid B_{j}^{i}, j \in \alpha\right) \\
H\left(T_{A^{i}}\left(C^{i}\right) \mid T_{A^{i}}\left(B_{j}^{i}\right), j \in \alpha\right) & =0 \\
\lim _{i \rightarrow \infty} \frac{1}{k(i)} H\left(T_{A^{i}}\left(B_{j}^{i}\right), j \in \beta\right) & =\lim _{i \rightarrow \infty} \frac{1}{k(i)} H\left(B_{j}^{i}, j \in \beta\right) .
\end{aligned}
$$

Proof: It is easy to pick a subspace $A$ of $C$ such that $H(A)=H\left(C \mid B_{j}, j \in \alpha\right)$ and that $A$ and $\left\langle B_{j}, j \in \alpha\right\rangle$ together span $\left\langle C, B_{j}, j \in \alpha\right\rangle$. Then it is straightforward to prove that $H\left(T_{A}(C) \mid T_{A}\left(B_{j}\right), j \in \alpha\right)=0$, which proves the first part of the lemma. 
Similarly, for each $i$, there exists a subspace $A^{i}$ such that $H\left(A^{i}\right)=H\left(C^{i} \mid B_{j}^{i}, j \in \alpha\right)$, and $H\left(T_{A^{i}}\left(C^{i}\right) \mid T_{A^{i}}\left(B_{j}^{i}\right), j \in \alpha\right)=0$. By Lemma 2, for any $\beta$

$$
H\left(B_{\beta}^{i}\right)-H\left(A^{i}\right) \leq H\left(T_{A}\left(B_{j}^{i}\right), j \in \beta\right) \leq H\left(B_{\beta}^{i}\right) .
$$

The remaining part of the lemma then follows as $\lim _{i \rightarrow \infty} \frac{1}{k(i)} H\left(A^{i}\right)=0$.

Lemma 5: Let $\left\{B_{1}, \ldots, B_{n}\right\}$ be a collection of subspaces and $\beta \subseteq \mathcal{N}$. Then there exists a subspace $A$ such that

$$
\begin{aligned}
H\left(T_{A}\left(B_{j}\right)\right) & =H\left(B_{j} \mid B_{\beta \backslash j}\right), \forall j \in \beta, \\
H\left(T_{A}\left(B_{j}\right), j \in \beta\right) & =\sum_{j \in \beta} H\left(T_{A}\left(B_{j}\right)\right), \\
H(A) & =H\left(B_{\beta}\right)-\sum_{j \in \beta} H\left(B_{j} \mid B_{\beta \backslash j}\right) .
\end{aligned}
$$

Furthermore, for a sequence of subspaces $\left\{B_{1}^{i}, \ldots, B_{n}^{i}\right\}_{i=1}^{\infty}$ and $k(i)>0$ such that

$$
\lim _{i \rightarrow \infty} \frac{1}{k(i)}\left(H\left(B_{\beta}^{i}\right)-\sum_{j \in \beta} H\left(B_{j}^{i} \mid B_{\beta \backslash j}^{i}\right)\right)=0 .
$$

there exists a sequence of subspaces $A^{i}$ such that for all $\alpha \subseteq \mathcal{N}$,

$$
\begin{array}{r}
\sum_{j \in \beta} H\left(T_{A^{i}}\left(B_{j}^{i}\right)\right)=H\left(T_{A^{i}}\left(B_{j}^{i}\right), j \in \beta\right) \\
\lim _{i \rightarrow \infty} \frac{1}{k(i)} H\left(T_{A^{i}}\left(B_{j}^{i}\right), j \in \alpha\right)=\lim _{i \rightarrow \infty} \frac{1}{k(i)} H\left(B_{\alpha}^{i}\right) .
\end{array}
$$

Proof: Define $A$ as the minimal subspace containing $B_{i} \cap\left\langle B_{j}, j \in \beta \backslash i\right\rangle$ for $i \in \beta$. Then it is straightforward to prove that for all $i, A$ is a subspace of $\left\langle B_{j}, j \in \beta \backslash i\right\rangle$ and hence $H\left(A \mid B_{j}, j \in \beta \backslash i\right)=0$. Similarly, for all $i, B_{i} \cap A=B_{i} \cap\left\langle B_{j}, j \in \beta \backslash i\right\rangle$ and hence $I\left(B_{i} ; A\right)=$ $I\left(B_{i} ; B_{j}, j \in \beta \backslash i\right)$ and

$$
H\left(B_{1}, \ldots, B_{n} \mid A\right)=\sum_{i=1}^{n} H\left(B_{i} \mid A\right)=H\left(B_{i} \mid B_{\beta \backslash i}\right)
$$

Consequently, (49)-(51) holds. The remaining part of the lemma be proved similarly as in Lemma 4.

The final ingredients that we require are the following results from [15]. Although these results were originally stated in terms of linear network coding capacity, we can restate them purely in terms of rank inequalities as follows. 
Theorem 7 (Dougherty, Freiling, Zeger): Suppose that $\mathcal{X}_{1}=\left\{Y_{1}, Y_{2}, Y_{3}, W_{1}, \ldots, W_{4}\right\}$ is a collection of vector subspaces over a finite field of odd characteristic. If the resulting polymatroid satisfies (44) and (45), then [15, Theorem IV.3]

$$
\frac{\min _{i=1,2,3} H\left(Y_{i}\right)}{\max _{j=1,2,3,4} H\left(W_{j}\right)} \leq \frac{4}{5} .
$$

Similarly, suppose that $\mathcal{X}_{2}=\left\{Z_{1}, \ldots, Z_{5}, V_{1}, \ldots, V_{8}\right\}$ is a collection of vector subspaces over a finite field of even characteristic. If $\mathcal{X}_{2}$ satisfies (46) and (47), then [15, Theorem IV.4]

$$
\frac{\min _{i=1, \ldots, 5} H\left(Z_{i}\right)}{\max _{j=1, \ldots, 8} H\left(V_{j}\right)} \leq \frac{10}{11} \text {. }
$$

Proof: [Proof of Theorem 6] Suppose to the contrary that $\left(\mathcal{X}, \Phi_{2}\right)$ is almost even representable. Then by definition there exists a sequence of even representable polymatroids $\left\{\left(\mathcal{X}, \mathbf{g}_{i}\right)\right\}_{i=1}^{\infty}$ and positive constants $d_{i}$ such that $\lim _{i \rightarrow \infty} d_{i} \mathbf{g}_{i}=\Phi_{2}$. While $\left(\mathcal{X}, \Phi_{2}\right)$ satisfies (46) and (47), these constraints may not be satisfied by $\left(\mathcal{X}, \mathbf{g}_{i}\right)$ in general. However, we can use Lemmas 4 and 5 to construct from $\left\{\left(\mathcal{X}, \mathbf{g}_{i}\right)\right\}_{i=1}^{\infty}$ another sequence of even representable polymatroids $\left\{\left(\mathcal{X}, \mathbf{g}_{i}^{\prime}\right)\right\}_{i=1}^{\infty}$ such that $\mathbf{g}_{i}^{\prime}$ satisfies (46) and (47), and $\lim _{i \rightarrow \infty} d_{i} \mathbf{g}_{i}^{\prime}=\lim _{i \rightarrow \infty} d_{i} \mathbf{g}_{i}=\Phi_{2}$. As such,

$$
\lim _{i \rightarrow \infty} \frac{\min _{k=1, \ldots, 5} \mathbf{g}_{i}^{\prime}\left(Z_{k}\right)}{\max _{k=1, \ldots, 8} \mathbf{g}_{i}^{\prime}\left(V_{k}\right)}=\frac{\min _{i=1, \ldots, 5} \Phi_{2}\left(Z_{k}\right)}{\max _{k=1, \ldots, 8} \Phi_{2}\left(V_{k}\right)} \stackrel{(a)}{=} 1,
$$

where (a) follows from connectivity of $\left(\mathcal{X}, \Phi_{2}\right)$. The existence of such a sequence $\left\{\left(\mathcal{X}, d_{i} \mathbf{g}_{i}^{\prime}\right)\right\}_{i=1}^{\infty}$ contradicts Theorem 7 which proved that the limit (52) is bounded above by $10 / 11$. Thus $\left(\mathcal{X}, \Phi_{2}\right)$ cannot be almost even representable. Using the same argument, we can also prove that $\left(\mathcal{X}, \Phi_{1}\right)$ is not almost odd representable.

\section{InSUFFICIENCY OF ALl KNOWN RANK INEQUALITIES}

In Section III], we constructed $(\mathcal{X}, \Phi) \in \overline{\operatorname{con}}(\Upsilon[\mathcal{X}])$ such that its $\epsilon$-pertubation $\left(\mathcal{X}, \Phi^{\epsilon}\right) \notin$ $\overline{\mathrm{con}}(\Upsilon[\mathcal{X}])$. Theorem 1 proved the existence of new subspace rank inequalities that are not implied by Ingleton's inequalities. This was achieved by showing that $\left(\mathcal{X}, \Phi^{\epsilon}\right)$ is Ingletonian. In the following, we will give another proof for Theorem 11. This alternative proof demonstrates the kind of difficulties one may face when characterizing representable polymatroids. Finally, we will generalize our main result to show that the newly discovered DFZ inequalities are also insufficient. 
Proof: [Alternative proof of Theorem 1] In [8] and [16], all the extreme vectors $\overline{\operatorname{con}}(\Upsilon[\mathcal{X}])$ are identified for $|\mathcal{X}|=4$. It can be easily verified that all of the associated rank functions are ternary representable. Hence, every vector in $\overline{\operatorname{con}}(\Upsilon[\mathcal{X}])$ is almost representable. By Theorem 3, its $\epsilon$-perturbed counterpart is almost representable.

Now, consider any Ingletonian polymatroid $(\mathcal{X}, \mathbf{h})$ and its $\epsilon$-perturbation $(\mathcal{X}, \mathbf{g})$ defined as in (8). For any subsets $\mathcal{V}_{1}, \ldots, \mathcal{V}_{4} \subseteq \mathcal{X}, \mathbf{h}$ induces a polymatroid $\left(\mathcal{N}_{4}, \mathbf{h}^{\prime}\right)$ via $\mathbf{h}^{\prime}(\alpha) \triangleq \mathbf{h}\left(\mathcal{V}_{i}, i \in\right.$ $\alpha)$. Clearly, $\mathbf{h}^{\prime}$ is also Ingletonian, since for any subsets $\alpha_{1}, \ldots, \alpha_{4} \subseteq \mathcal{N}_{4}, J_{\mathbf{h}^{\prime}}\left(\alpha_{1}, \ldots, \alpha_{4}\right)=$ $J_{\mathbf{h}}\left(\mathcal{V}_{\alpha_{1}}, \ldots, \mathcal{V}_{\alpha_{4}}\right)$. Hence, $\mathbf{h}^{\prime}$ is also almost representable. By Theorem 3 , its perturbation is also almost representable.

Now, if $\mathbf{h}(\mathcal{X})-\epsilon \geq \mathbf{h}^{\prime}\left(\mathcal{N}_{4}\right)$, then clearly

$$
\mathbf{g}\left(\mathcal{V}_{i}, i \in \alpha\right)=\mathbf{h}\left(\mathcal{V}_{i}, i \in \alpha\right)
$$

for all $\alpha$. Then $J_{\mathbf{g}}\left(\mathcal{V}_{\alpha_{1}}, \ldots, \mathcal{V}_{\alpha_{4}}\right)=J_{\mathbf{h}}\left(\mathcal{V}_{\alpha_{1}}, \ldots, \mathcal{V}_{\alpha_{4}}\right) \geq 0$.

On the other hand, suppose that $\mathbf{h}(\mathcal{X})-\epsilon \leq \mathbf{h}^{\prime}\left(\mathcal{N}_{4}\right)$. Then

$$
\begin{aligned}
\mathbf{g}\left(\mathcal{V}_{i}, i \in \alpha\right) & =\min \left(\mathbf{h}\left(\mathcal{V}_{i}, i \in \alpha\right), \mathbf{h}(\mathcal{X})-\epsilon\right) \\
& =\min \left(\mathbf{h}^{\prime}(\alpha), \mathbf{h}(\mathcal{X})-\epsilon\right) \\
& =\min \left(\mathbf{h}^{\prime}(\alpha), \mathbf{h}^{\prime}\left(\mathcal{N}_{4}\right)+\mathbf{h}(\mathcal{X})-\mathbf{h}^{\prime}\left(\mathcal{N}_{4}\right)-\epsilon\right) \\
& =\min \left(\mathbf{h}^{\prime}(\alpha), \mathbf{h}^{\prime}\left(\mathcal{N}_{4}\right)-\nu\right)
\end{aligned}
$$

where $\nu \triangleq \mathbf{h}^{\prime}\left(\mathcal{N}_{4}\right)+\epsilon-\mathbf{h}(\mathcal{X})$. Let

$$
\mathbf{g}^{\prime}(\alpha) \triangleq \min \left(\mathbf{h}^{\prime}(\alpha), \mathbf{h}^{\prime}\left(\mathcal{N}_{4}\right)-\nu\right) .
$$

Then $\mathbf{g}^{\prime}$ is almost representable and hence $J_{\mathbf{g}}\left(\mathcal{V}_{\alpha_{1}}, \ldots, \mathcal{V}_{\alpha_{4}}\right)=J_{\mathbf{g}^{\prime}}\left(\alpha_{1}, \ldots, \alpha_{4}\right) \geq 0$.

Theorem 8 (Generalization): Suppose $|\mathcal{Y}|=n$ and that all vectors $\mathrm{g} \in \overline{\operatorname{con}}(\Upsilon[\mathcal{Y}])$ are almost representable. Consider any valid subspace rank inequality of the form

$$
\sum_{\alpha \subseteq \mathcal{N}_{n}} c_{\alpha} H\left(\mathcal{V}_{i}, i \in \alpha\right) \geq 0
$$

where $\mathcal{V}_{i} \subseteq \mathcal{Y}$. Then for any cc-representable polymatroid $(\mathcal{X}, \mathbf{h})$ (i.e., $\mathbf{h} \in \overline{\operatorname{con}}(\Upsilon[\mathcal{X}])$ ), its perturbed counterpart will satisfy the inequality (58). Consequently $\left(\mathcal{X}, \Phi^{\epsilon}\right)$ in Section $\nabla$ will 
satisfy (58) and hence any inequalities involving no more than $n$ subsets are insufficient to characterize $\overline{\operatorname{con}}(\Upsilon[\mathcal{X}])$ in general.

Proof: The proof is similar to the alternative proof for Theorem 5 ,

The newly discovered inequalities [12] were shown to be sufficient to characterize $\overline{\operatorname{con}}(\Upsilon[\mathcal{X}])$ when $|\mathcal{X}| \leq 5$. In fact, it was further proved that all the extreme vectors of the cone $\overline{\operatorname{con}}(\Upsilon[\mathcal{X}])$ are $q$-representable, for sufficiently large $q$. As a result, every vector in $\overline{\operatorname{con}}(\Upsilon[\mathcal{X}])$ will be almost representable. By Theorem 8 , these newly discovered inequalities are insufficient to characterize $\overline{\operatorname{con}}(\Upsilon[\mathcal{X}])$ in general.

\section{CONCLUSION}

A complete characterization of representable polymatroids has been open for years. This problem is fundamental in nature and is intimately related to the information thoeretic problem of the characterization of transmission throughput in networks with linear network coding. Until quite recently it was not know whether Ingleton's inequalities are sufficient to characterize all representable polymatroids. In this paper, we have constructed an Ingletonian polymatroid that satisfies all known (Ingleton and Dougherty-Freiling-Zeger) subspace rank inequalities. As a result, there are inequalities remaining to be discovered. While our approach does not suggest how to construct these new inequalities, it at least demonstrates some of the difficulties of the problem.

\section{ACKNOWLEDGMENT}

This work was supported by the Australian Government under ARC grant DP0880223.

\section{REFERENCES}

[1] R. Ahlswede, N. Cai, S.-Y. R. Li, and R. W. Yeung, "Network information flow," IEEE Trans. Inform. Theory, vol. 46, no. 4, pp. 1204-1216, July 2000.

[2] S.-Y. R. Li, R. Yeung, and N. Cai, "Linear network coding," IEEE Trans. Inform. Theory, vol. 49, no. 2, pp. 371-381, Feb. 2003.

[3] R. Yeung, A First Course in Information Theory. Kluwer Academic/Plenum Publisher, 2002.

[4] X. Yan, R. W. Yeung, and Z. Zhang, "The capacity region for multi-source multi-sink network coding," submitted to IEEE Trans. Inform. Theory, 2009. 
[5] T. H. Chan, "Capacity regions for linear and abelian network code," in NETCOD, San Diego, USA, 2007.

[6] T. H. Chan and A. Grant, "Dualities between entropy functions and network codes," IEEE Trans. Inform. Theory, pp. 4470-4487, Oct. 2008.

[7] C. E. Shannon, "A mathematical theory of communication,” Bell Syst. Tech. J., vol. 27, pp. 379-423, 623-656, 1948.

[8] Z. Zhang and R. W. Yeung, "On the characterization of entropy function via information inequalities," IEEE Trans. Inform. Theory, vol. 44, pp. pp. 1440-1452, 1998.

[9] R. Dougherty, C. Freiling, and K. Zeger, "Six new non-shannon information inequalities," in IEEE Int. Symp. Inform. Theory, July 2006, pp. 233-236.

[10] F. Matus, "Infinitely many information inequalities," in Proceedings ISIT 2007, June 2007, (Nice, France), 2007, pp. 41-44.

[11] A. W. Ingleton, "Representation of matroids.” London: Academic Press, 1971, pp. 149-167.

[12] R. Dougherty, C. Freiling, and K. Zeger, "Non-shannon entropy inequalities and linear rank inequalities," Workshop on Applications of Matroid Theory and Combinatorial Optimization to Information and Coding Theory, August 2009. [Online]. Available: http://robson.birs.ca/ 09w5103/dougherty_09w5103_talk2.pdf

[13] J. Oxley, Matroid Theory. Oxford University Press, 1992.

[14] R. Yeung, “A framework for linear information inequalities,” IEEE Trans. Inform. Theory, vol. 43, no. 6, pp. 1924-1934, Nov. 1997.

[15] R. Dougherty, C. Freiling, and K. Zeger, "Insufficiency of linear coding in network information flow," IEEE Trans. Inform. Theory, vol. 51, no. 8, pp. 2745-2759, Aug. 2005.

[16] D. Hammer, A. Romashchenko, A. Shen, and N. Vereshchagin, "Inequalities for shannon entropy and kolmogorov complexity," Journal of Computer and System Sciences, vol. 60, pp. 442-464, 2000. 